Article

\title{
Porcine Parvovirus 7: Evolutionary Dynamics and Identification of Epitopes toward Vaccine Design
}

\author{
Dongliang Wang, Jinhui Mai, Yi Yang $\$ and Naidong Wang * \\ Hunan Provincial Key Laboratory of Protein Engineering in Animal Vaccines, Laboratory of Functional \\ Proteomics (LFP), Research Center of Reverse Vaccinology (RCRV), College of Veterinary Medicine, \\ Hunan Agricultural University, Changsha 410128, China; dongliangwang@stu.hunau.edu.cn (D.W.); \\ jinhuimai@stu.hunau.edu.cn (J.M.); yiyang@hunau.edu.cn (Y.Y.) \\ * Correspondence: naidongwang@hunau.edu.cn
}

Received: 5 June 2020; Accepted: 1 July 2020; Published: 5 July 2020

\begin{abstract}
Porcine parvovirus 7 (PPV7) belonging to the genus Chapparvovirus in the family Parvoviridae, has been identified in the USA, Sweden, Poland, China, South Korea and Brazil. Our objective was to determine the phylogeny, estimate the time of origin and evolutionary dynamics of PPV7, and use computer-based immune-informatics to assess potential epitopes of its Cap, the main antigenic viral protein, for vaccines or serology. Regarding evolutionary dynamics, PPV7 had 2 major clades, both of which possibly had a common ancestor in 2004. Furthermore, PPV7 strains from China were the most likely ancestral strains. The nucleotide substitution rates of NS1 and Cap genes were $8.01 \times 10^{-4}$ and $2.19 \times 10^{-3}$ per site per year, respectively, which were higher than those reported for PPV1-4. The antigenic profiles of PPV7 Cap were revealed and there were indications that PPV7 used antigenic shift to escape from the host's immune surveillance. Linear B cell epitopes and CD8 T cell epitopes of Cap with good antigenic potential were identified in silico; these conserved B cell epitopes may be candidates for the PPV7 vaccine or for the development of serological diagnostic methods.
\end{abstract}

Keywords: PPV7; Cap; B cell and T cell epitopes; vaccine; evolution

\section{Introduction}

The family Parvoviridae contains two subfamilies: Parvovirinae and Densovirinae, whose hosts are vertebrates and arthropods, respectively [1]. The subfamily Parvovirinae is further divided into nine genera: Tetraparvovirus, Copiparvovirus, Erythroparvovirus, Dependoparvovirus, Aveparvovirus, Bocaparvovirus, Amdoparvovirus, Protoparvovirus and Chapparvovirus [1,2]. To date, seven genotypes of porcine parvoviruses (PPV) have been discovered in pigs, and belong to four genera: Protoparvovirus (PPV1), Tetraparvovirus (PPV2-3), Copiparvovirus (PPV4-6) and Chapparvovirus (PPV7), based on the similarity of non-structural protein 1 (NS1) [2,3]. PPV1, first identified in 1965 in Germany [4], is a major cause of reproductive failure in pigs, characterized by mummified fetuses, infertility, early embryonic death, stillbirths and delayed returns to the estrus [5,6]. Subsequently, six other PPV genotypes (PPV2-PPV7) were identified using novel techniques, including next-generation sequencing [2,7-11].

In 2016, PPV7 was first identified by the metagenomic sequencing of rectal swab samples from pigs in the USA [2]. Subsequently, PPV7 infections were reported in Sweden, Poland, China, South Korea and Brazil [12-19]. PPV7 is a small, single-stranded linear and non-enveloped DNA virus with a genome of $\sim 4 \mathrm{~kb}$ that contains two major open reading frames (ORFs): ORF1 encodes non-structural protein 1 (NS1) responsible for viral replication, and ORF2 encodes the major structural capsid protein (Cap) or VP2 protein [2]. PPV Cap is also the major antigenic component, with an important role in eliciting neutralizing antibodies against viral infection [20]. In addition, it is a prospective antigen for subunit vaccine design and serological diagnosis. A vaccine based on the Cap could elicit antibodies to 
neutralize virus infection by blocking virus entry [21]. Thus, the Cap could be regarded as an effective antigenic component for PPV7 vaccine design and development. Currently, there is no, or very limited, information about its antigenic structure and immunogenic profiles, thus, the immune profiles of PPV7 Cap are required to develop PPV7 Cap-based vaccines against PPV7 infection.

Despite limited knowledge regarding PPV7 pathogenicity, its presence in aborted pig fetuses suggests that it causes reproductive failure [17]. Co-infections are more frequent than single infection in swine herd; multiple infectious pathogens such as PCV2 and PPV can impact the outcome of respiratory infections and deserve [22]. In addition, co-infections with PPV7 and PCV2 were recently reported [23], although whether this co-infection enhanced the severity of porcine circovirus associated diseases (PCVADs) has yet to be determined. To better understand the molecular evolution and genetic diversity of this newly emerging PPV7, we analyzed the phylogeny and estimated the time of origin and evolutionary dynamics of PPV7. Importantly, computer-based immune-informatics were used to assess the potential B cell and CD8 T cell epitopes of the PPV7 Cap, which will greatly facilitate the development of new generation vaccines against PPV7 infection and contribute to the development of effective serological diagnostic methods.

\section{Materials and Methods}

\subsection{Sequence Datasets}

A total of 45 complete (or partial) genomes, and 59 NS1 and Cap complete coding sequences, were collected from GenBank to perform phylogenetic analysis. Detailed information of PPV7 and reference Parvoviridae sequences are summarized in Tables S1 and S2, respectively.

\subsection{Multiple Sequence Alignment}

NS1 and Cap amino acid sequences were derived from 59 PPV7 genomes. Sequence identity was analyzed and aligned with the Clustal W method of the MegAlign program of DNASTAR, version 7.10 (Lasergene) (DNASTAR, Inc., Wisconsin, USA). Amino acid sequences were aligned with the ESPript 3.0 online tool (ESPript 3.0, http://espript.ibcp.fr/ESPript/ESPript/, 4 July 2020).

\subsection{Phylogenetic and Evolution Dynamic Analysis}

Sequences were aligned with Clustal $W$, implemented in MEGA 7 software [24]. A maximum-likelihood (ML) tree was constructed with NS1 and Cap amino acid sequences, using MEGA 7 with the Jones-Taylor-Thornton (JTT) model and 1000 bootstrap replicates. The $p$-distance method was used to reconstruct a neighbor joining (NJ) tree with 1000 bootstrap replicates.

The most recent common ancestor (tMRCA) and rates of nucleotide substitutions per site per year were estimated using the Bayesian Markov chain Monte Carlo (MCMC) method within the BEAST package (Version 1.10.4) [25]. A general time reversible (GTR) substitution model with a proportion of gamma and invariant distributed rate heterogeneity $(G T R+G+I)$ with an uncorrelated relaxed lognormal molecular clock was selected. Chain length for the run was $1 \times 10^{8}$ generations, with sampling at every 10,000 generations. After removing the initial 10\% of samples as burn-in, only runs with an estimate sample size (ESS) > 200 were accepted, based on the software Tracer (v1.7.1) (Tracer, http://tree.bio.ed.ac.uk/software/tracer/, 4 July 2020). A maximum clade credibility (MCC) tree was reconstructed with TreeAnnotator (v1.10.4) and displayed in Figtree (v1.4.4) (Figtree, http://tree.bio.ed.ac.uk/software/figtree/, 4 July 2020).

\subsection{Selection Pressures Analysis}

The detection of selected NS1 and Cap coding sequences of PPV7 was performed using DATAMONKEY (Datamonkey, http://www.datamonkey.org/, 4 July 2020). Positive selected sites were detected using 4 algorithms, including fixed effects likelihood (FEL), single-likelihood ancestor counting (SLAC), fast unconstrained Bayesian approximation (FUBAR) and mixed effects model 
of evolution (MEME) [26-28]. A site was considered as a positive selection position only if it was identified by at least 2 algorithms, and with $p<0.1$ in SLAC, $p<0.05$ in FEL and MEME and posterior probability $>0.9$ in FUBAR were considered significant. The selection pressure analysis of genomes was determined with MEGA7 software by calculating the differences between non-synonymous (dN) and synonymous substitution (dS) rates, for the aligned genes. The calculated value of $\mathrm{dN}-\mathrm{dS}$ was used to evaluate selection pressure $(\mathrm{dN}-\mathrm{dS}>0$ : positive selection; $\mathrm{dN}-\mathrm{dS}<0$ : purifying selection; and $\mathrm{dN}-\mathrm{dS}=0$ : neutral selection) [29].

\subsection{Structural Analysis}

The physicochemical properties of PPV7 Cap were assessed with the ProtParam webserver (ProtParam, https://web.expasy.org/protparam/, 4 July 2020). The secondary structure of PPV7 Cap was predicted with PSIPRED [30].

\subsection{B Cell Epitope Prediction}

Linear B cell epitopes of the PPV7 Cap were predicted using the BepiPred 2.0 online tool in IEDB (Immune Epitope Database) (BepiPred 2.0, http://www.iedb.org/, 4 July 2020), with a threshold of 0.55 (corresponding specificity $>0.817$ and sensitivity $<0.292$ ); only epitopes with $>7$ residues were considered for subsequent antigenicity analysis. Antigenicity testing was done with the VaxiJen v2.0 server online tool [31] (VaxiJen v2.0, http://www.ddg-pharmfac.net/vaxijen/VaxiJen/VaxiJen.html, 4 July 2020).

\subsection{CD8 T Cell Epitope Prediction}

For CD8 T cell epitopes prediction, 3 porcine MHC-I molecules SLA-1*04:01, SLA-2*04:01 and SLA-3*04:01 [32-34] were selected, based on the IEDB recommended 2.22 algorithm in IEDB, with a peptide size of 9 residues. Higher scoring peptides (rank $\leq 2 \%$ ) (threshold $0.5 \%$ and $2.0 \%$ rank for strong and weak binders, respectively) based on the prediction were chosen for subsequent immunogenicity evaluation by the VaxiJen v2.0 server.

\subsection{Peptide Modelling and Molecular Docking}

The 3D structures of all peptides were modelled with the PEP-FOLD3 online server [35]. All peptides were docked to correspond to SLA-2*04:02 (PDB ID: 6A6H) and SLA-3*04:01 (PDB ID: 5H94), using the PatchDock rigid-body docking server, using the defined threshold [36]. Based on geometry docking algorithm in PatchDock, the docking transformation with good molecular shape complementarity was selected, and docked complexes were refined with the FireDock server [37,38]. Complexes with high global energy, attractive Vander Waal (vdW) energy and hydrogen bonding energy were used for subsequent analyses. Protein peptide connections were examined with the LigPlus tool and analyzed with Pymol.

\section{Results}

\subsection{Construction of Phylogenetic Tree and Evolution Dynamic Analysis}

To better understand genetic relationships between PPV7 and other strains of Parvoviridae, a phylogenetic tree was constructed, using the maximum likelihood (ML) and neighbor joining (NJ) methods, based on the NS1 and Cap amino acid sequences, respectively. According to phylogenetic analyses, all PPV7 strains were located in a branch belonging to the Chapparvovirus genus (Figure 1 and Figure S1). Additionally, PPV7 was more genetically close to PPV1, compared to the PPV strains among the other genotypes (Figure 1). Furthermore, the maximum clade credibility (MCC) tree was reconstructed with 45 complete PPV7 genomes. It appeared that PPV7 strains may have had a common ancestor in 2004 (95\% highest posterior density (HPD): 1986-2014); furthermore, the PPV7 strains from China were the most likely ancestral strains, based on currently available sequences (Figure 2). 
A

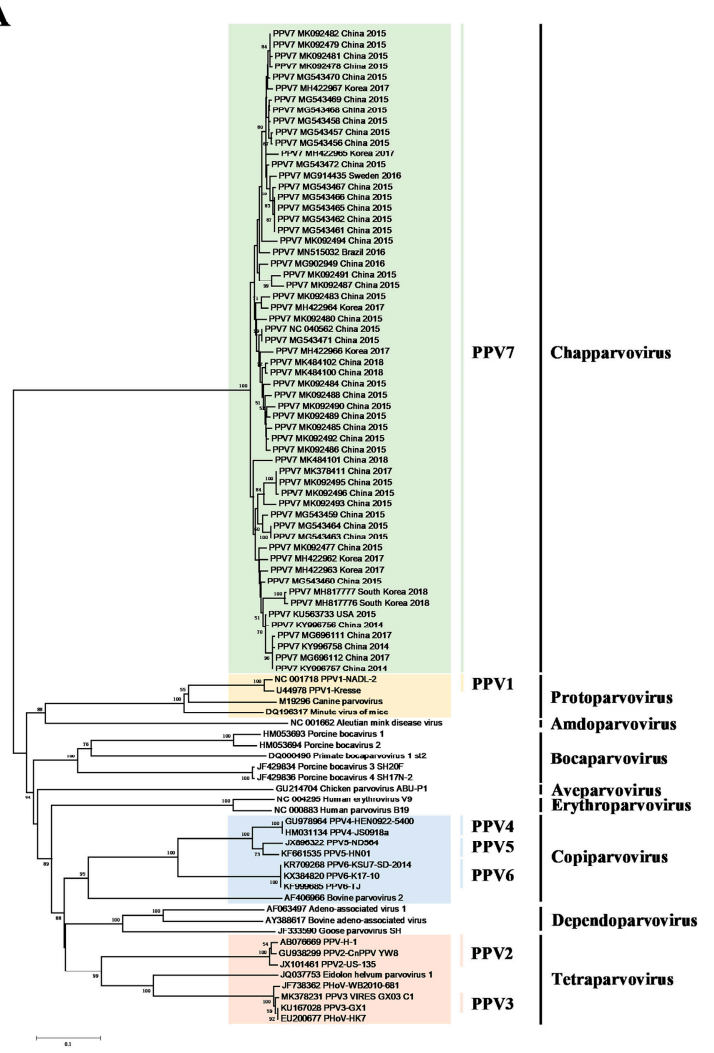

B

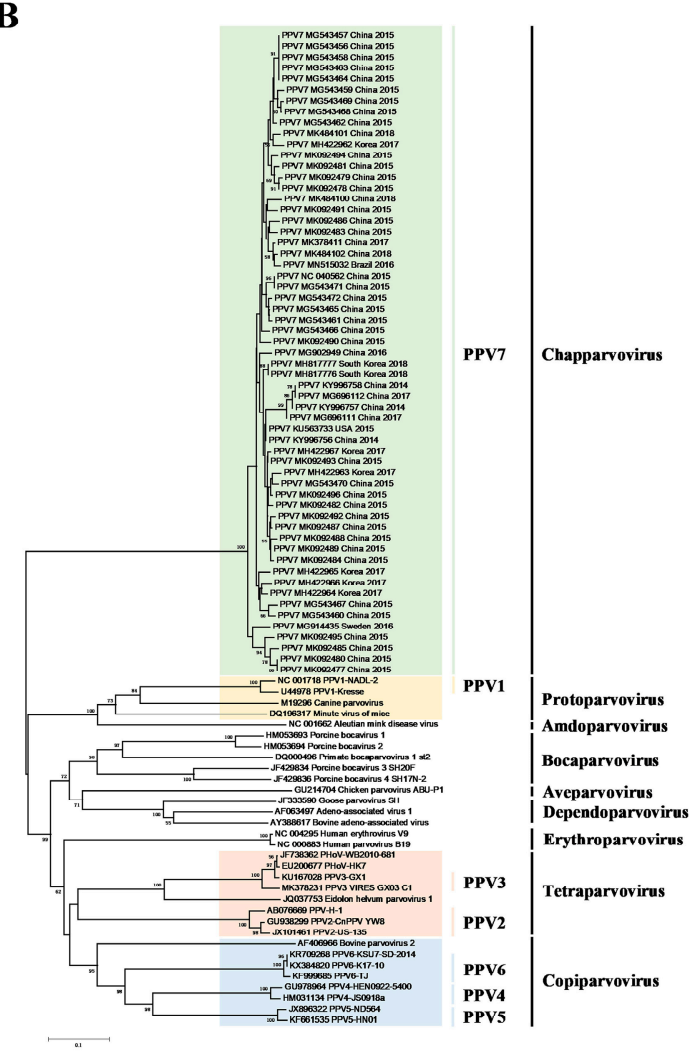

Figure 1. Phylogenetic analysis of viruses in the Parvoviridae family. Trees were constructed based on the NS1 (A) and Cap (B) amino acid sequences by the neighbor joining (NJ) phylogenetic method, using the $\mathrm{p}$-distance model with 1000 bootstrap replicates and bootstrap $>50 \%$.

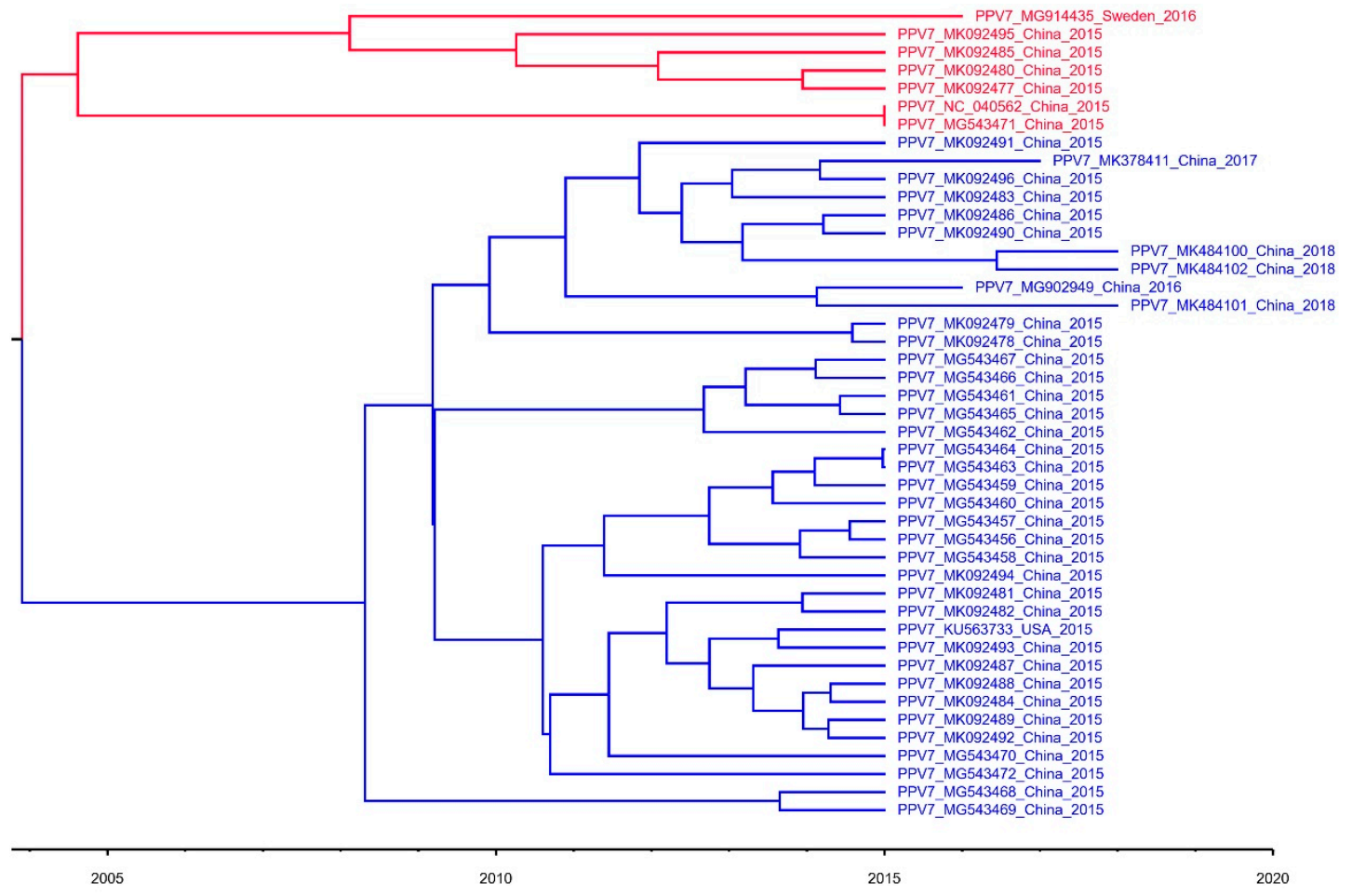

Figure 2. Maximum clade credibility (MCC) tree for the complete genome sequences of PPV7. The tree contained 2 major clades: Clade 1 (red) and Clade 2 (blue). 
The evolutionary rates of the NS1 and Cap genes of PPV7 were estimated. The mean evolutionary rates of the NS1 and Cap genes were $8.01 \times 10^{-4}$ per site per year (95\% HPD: $3.67 \times 10^{-6}-1.9 \times 10^{-3}$ ) and $2.19 \times 10^{-3}$ per site per year $\left(95 \%\right.$ HPD: $\left.1.28 \times 10^{-4}-5.06 \times 10^{-3}\right)$, respectively.

\subsection{Selection Pressures Analysis}

Five sites $(24,106,158,270$ and 446) among Cap were confirmed by all 4 methods to be under positive selection. Furthermore, 2 sites (195 and 441) were also confirmed to be under positive selection in Cap by at least 2 methods with $p<0.05$ by FEL and MEME, $p<0.1$ by SLAC, and a posterior probability $>0.9$ by FUBAR (Table 1). In addition, 8 NS1 sites $(69,342,426,465,474,498,525$ and 603) were detected as positive selection positions by all 4 methods, whereas the other 9 sites $(27,83,376,500$, $547,549,550,579$ and 618) were detected as positive selection positions by at least 2 methods (Table 2). The overall mean differences of $\mathrm{dN}-\mathrm{dS}$ were -4.122 for Cap gene and -15.371 for NS1 gene, indicating that both Cap and NS1 were under purifying selection.

Table 1. Selection analysis of PPV7 Cap coding sequences.

\begin{tabular}{|c|c|c|c|c|c|c|c|c|c|c|c|}
\hline \multicolumn{3}{|c|}{ FEL } & \multicolumn{3}{|c|}{ SLAC } & \multicolumn{3}{|c|}{ FUBAR } & \multicolumn{3}{|c|}{ MEME } \\
\hline Site & $\mathrm{dN}-\mathrm{dS}$ & $p$-Value & Site & $\mathrm{dN}-\mathrm{dS}$ & $p$-Value & Site & $\mathrm{dN}-\mathrm{dS}$ & Post.Pro & Site & $\beta^{+}$ & $p$-Value \\
\hline 24 & 2.492 & 0.004 & 24 & 5.741 & 0.018 & 24 & 6.900 & 0.997 & 24 & 2.5 & 0.01 \\
\hline 106 & 2.157 & 0.023 & 106 & 4.943 & 0.028 & 106 & 5.706 & 0.979 & 106 & 24.21 & 0 \\
\hline \multirow[t]{2}{*}{158} & 1.552 & 0.033 & 158 & 3.652 & 0.098 & 158 & 3.244 & 0.972 & 158 & 1.55 & 0.05 \\
\hline & & & & & & 195 & 1.442 & 0.909 & 195 & 166.2 & 0 \\
\hline \multirow[t]{2}{*}{270} & 1.777 & 0.026 & 270 & 5.107 & 0.048 & 270 & 4.363 & 0.974 & 270 & 14.87 & 0 \\
\hline & & & & & & 441 & 2.926 & 0.944 & 441 & 18.72 & 0 \\
\hline 446 & 1.649 & 0.015 & 446 & 3.970 & 0.055 & 446 & 3.901 & 0.988 & 446 & 3.43 & 0.02 \\
\hline
\end{tabular}

Table 2. Selection analysis of PPV7 NS1 coding sequences.

\begin{tabular}{|c|c|c|c|c|c|c|c|c|c|c|c|}
\hline \multicolumn{3}{|c|}{ FEL } & \multicolumn{3}{|c|}{ SLAC } & \multicolumn{3}{|c|}{ FUBAR } & \multicolumn{3}{|c|}{ MEME } \\
\hline Site & $\mathrm{dN}-\mathrm{dS}$ & $p$-Value & Site & dN-dS & $p$-Value & Site & dN-dS & Post.Pro & Site & $\beta^{+}$ & $p$-Value \\
\hline 27 & 1.519 & 0.016 & & & & 27 & 2.24 & 0.977 & 27 & 1.52 & 0.03 \\
\hline \multirow[t]{2}{*}{69} & 2.815 & 0.002 & 69 & 3.466 & 0.069 & 69 & 4.953 & 0.997 & 69 & 2.82 & 0 \\
\hline & & & & & & 83 & 2.627 & 0.96 & 83 & 148.66 & 0 \\
\hline \multirow[t]{2}{*}{342} & 5.477 & 0.01 & 342 & 8.331 & 0.005 & 342 & 12.563 & 0.993 & 342 & 173.79 & 0 \\
\hline & & & 376 & 4.566 & 0.066 & 376 & 5.16 & 0.965 & & & \\
\hline 426 & 8.215 & 0.005 & 426 & 10.973 & 0.002 & 426 & 17.289 & 0.999 & 426 & 40.1 & 0 \\
\hline 465 & 1.905 & 0.034 & 465 & 3.658 & 0.088 & 465 & 2.882 & 0.979 & 465 & 1.9 & 0.05 \\
\hline 474 & 4.118 & 0.019 & 474 & 5.486 & 0.026 & 474 & 7.406 & 0.983 & 474 & 4.12 & 0.03 \\
\hline \multirow[t]{2}{*}{498} & 4.261 & 0 & 498 & 5.326 & 0.031 & 498 & 8.12 & 1 & 498 & 4.25 & 0 \\
\hline & & & 500 & 5.817 & 0.028 & 500 & 2.963 & 0.956 & & & \\
\hline \multirow[t]{4}{*}{525} & 7.599 & 0.003 & 525 & 10.047 & 0.002 & 525 & 15.702 & 0.999 & 525 & 7.6 & 0 \\
\hline & & & 547 & 5.486 & 0.026 & 547 & 4.412 & 0.97 & & & \\
\hline & & & 549 & 4.267 & 0.059 & 549 & 2.533 & 0.956 & & & \\
\hline & & & 550 & 3.658 & 0.088 & 550 & 2.944 & 0.992 & 550 & 1.9 & 0.01 \\
\hline 579 & 2.081 & 0.01 & & & & 579 & 3.455 & 0.994 & 579 & 2.08 & 0.02 \\
\hline \multirow[t]{2}{*}{603} & 1.271 & 0.025 & 603 & 3.778 & 0.081 & 603 & 1.998 & 0.974 & 603 & 1.27 & 0.04 \\
\hline & & & 618 & 4.105 & 0.077 & 618 & 2.663 & 0.95 & & & \\
\hline
\end{tabular}

\subsection{Sequence and Structural Characteristics}

Sequence alignments revealed that PPV7 genomes (total 45 of isolates deposited in GenBank) exhibited 91.9-100\% nucleotide homology. Furthermore, PPV7 NS1 and Cap genes (total 59 of each deposited in GenBank) had 92.8-100\% and 85.7-100\% homology, respectively, and the derived amino acid sequence of both proteins shared $89.9-100 \%$ and $82.4-100 \%$ identity, respectively. The $\mathrm{Ca}^{2+}$ binding loop (YXGXG) was present in the Caps of PPV1, PPV2, PPV3 and PPV5 [1,10], and "YXGXR" was in PPV6 [11], but absent in PPV4. However, the potential $\mathrm{Ca}^{2+}$ binding loop was the ${ }^{267} \mathrm{YXGXXG}{ }^{272}$ motif in PPV7, and "269 GXX ${ }^{271 " ~(" 269} \mathrm{GPP}^{271 ")}$ were strictly conserved in PPV7 (Figure 3). Furthermore, compared to the catalytic motif (HDXXY) of the putative secretory phospholipase A2 (PLA2) in PPV5 [10], a similar motif ${ }^{300} \mathrm{HDXXN}{ }^{304}$ was present in PPV7, whereas a point mutation occurred at position 304 (N 304 Y) in the 59 of PPV7 Cap proteins (Figure 3). 

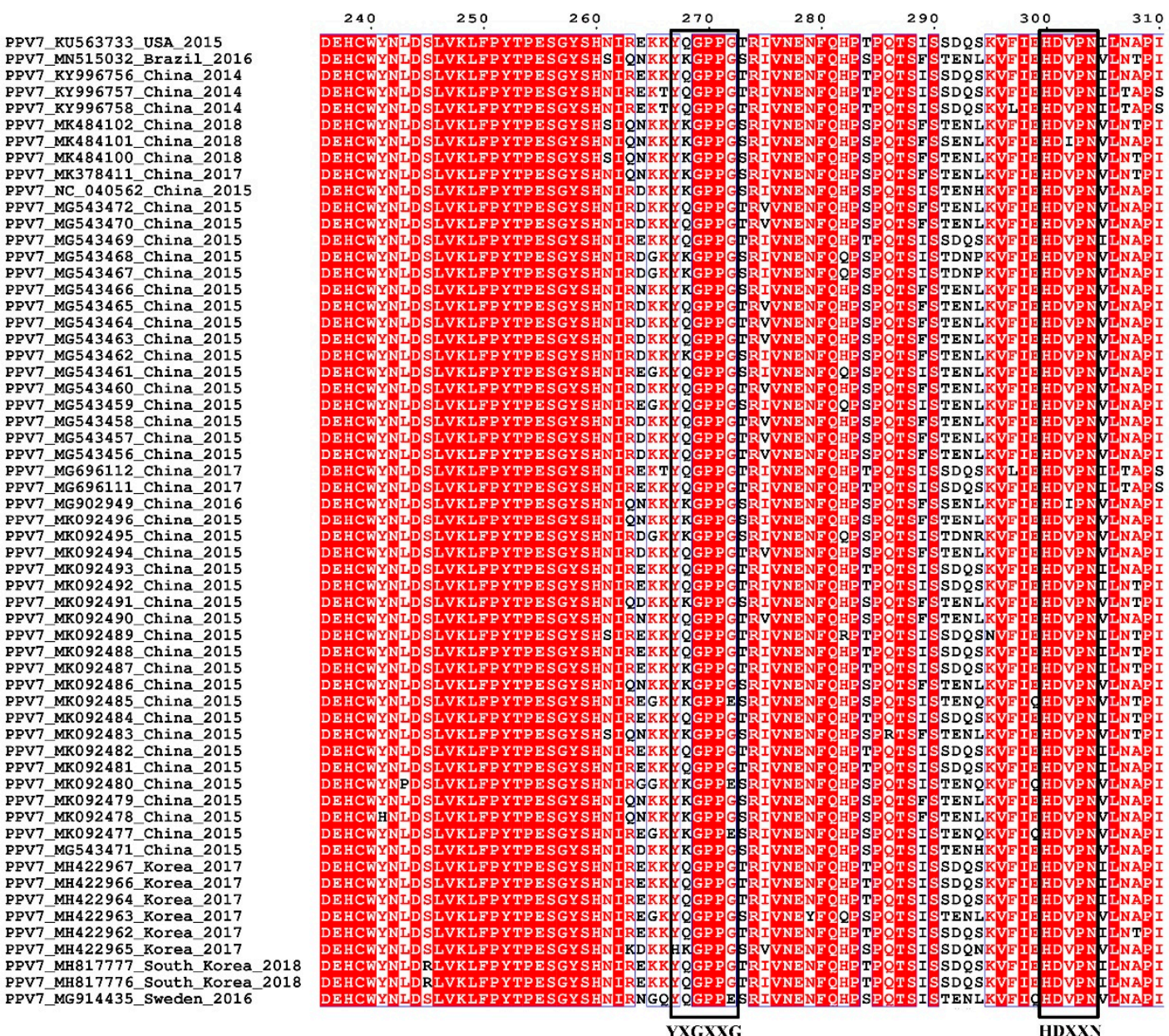

Figure 3. Sequence alignment of $\mathrm{Ca}^{2+}$ binding loop and putative secretory phospholipase A2 (PLA2) motif of PPV7. The conserved amino acids of the $\mathrm{Ca}^{2+}$ binding loop $\left({ }^{267}{ }^{2} X G X X G^{272}\right)$ and the catalytic residues $\left({ }^{300} \mathrm{HDXXN}{ }^{304}\right)$ are indicated at the bottom of the alignment.

A representative strain (KU563733) was used for a structural analysis of PPV7 Cap. The physicochemical properties of the PPV7 Cap, computed using the ProtParam tool, concluded that it contained $469 \mathrm{aa}$, with a molecular weight of $54,257.8 \mathrm{Da}$. The isoelectric point (PI) of this protein was 7.23 , indicating that it is positive in nature. Out of 469 residues, 42 (Asp + Glu) were negatively charged and 42 (Arg + Lys) were positively charged. The instability index of (II) was computed to be 38.58, indicating that it was a stable protein. The predicted aliphatic index was 56.78 and the grand average of hydropathicity (GRAVY) for the protein sequence was -0.695 . This protein contained 7483 atoms, and was described by the formula C2469H3639N647O714S14. The estimated half-life was $30 \mathrm{~h}$ (mammalian reticulocytes, in vitro), $>20 \mathrm{~h}$ (yeast, in vivo) and $>10 \mathrm{~h}$ (Escherichia coli, in vivo).

The secondary structure of the PPV7 Cap was analyzed by PSIPRED with $(24 \%)$ beta sheets, $(12 \%)$ helixes and (64\%) loops present in the structure (Figure S2). VaxiJen v2.0 was used to evaluate the antigenicity of the Cap. By setting the threshold at 0.4 for higher specificity, antigenicity score was 0.4426 for the Cap, implying an excellent antigenic potential.

\subsection{Linear B Cell Epitopes Prediction and Analysis of Cap}

In total, 10 potential linear B-cell epitopes were predicted with BepiPred 2.0, 6 of which were chosen for subsequent analyses based on antigenicity scores evaluated by VaxiJen v2.0 (Table 3). The prediction of the secondary structure and sequence alignment of PPV7 Caps suggested that amino acid mutations occurred predominantly in loops, and the six potential epitopes were all located in these loops (Figure S3). Among these 6 epitopes, epitopes C and E were highly conserved (with 98.3 
and 100\% identity, respectively) throughout all 59 isolates (Table 3 and Figure S3). The epitope C sequences (IQELRPGKN) in 58 isolates were identical, except that 2 mutations occurred in one isolate of PPV7-77 (IQELMPRKN). However, these 2 mutations in this isolate significantly decreased the antigenic score from 0.7908 to 0.2766 . The other 4 epitopes exhibited high variations among these 59 PPV7 isolates, with 1 positive selection site $(270 \mathrm{~K})$ and 2 sites $(441 \mathrm{~K}$ and $446 \mathrm{P})$ present in epitopes D and F, respectively. Generally, variations of the capsid surface, caused by residue mutations, may alter antigenic profiles, which results in the differences in cross-protective activities. Thus, the alteration of the PPV7 capsid antigenic profile is one of the strategies of PPV7 to adapt to the host during the virus evolution.

Table 3. Linear B cell epitopes prediction of PPV7 Cap using BepiPred 2.0.

\begin{tabular}{ccccccc}
\hline Epitope & Start & End & Sequence & Length & $\begin{array}{c}\text { Identity } \\
\text { (59 Isolates) }\end{array}$ & $\begin{array}{c}\text { Vaxijen v2.0 } \\
\text { Score }\end{array}$ \\
\hline A & 111 & 118 & YETGYHNW & 8 & $72.88 \%$ & 0.5888 \\
B & 135 & 150 & LVPKPTTATKEGVGNS & 16 & $18.64 \%$ & 0.4355 \\
C & 216 & 224 & IQELRPGKN & 9 & $98.31 \%$ & 0.7908 \\
D & 255 & 272 & ESGYSHNIREKKYQGPPG & 18 & $25.42 \%$ & 0.6235 \\
E & 385 & 391 & KRRSRMF & 7 & $100.00 \%$ & 1.6044 \\
F & 419 & 445 & TGGARRSWQARTRDTRDKEPQQPWYQW & 27 & $10.17 \%$ & 0.4966 \\
\hline
\end{tabular}

Further, we reviewed 8 linear B cell epitope regions of PPV1 Cap deposited in IEDB database, according to the corresponding peptide overlap (Table S3). Except for regions A and D, 6 of the 8 linear B cell epitope regions were mapped to the most exposed surface regions of the PPV1 capsid (Figure S4A). Comparison of the epitope sequences revealed that there were no homologous sequences between the PPV7 and PPV1 (Figure 4 ) due to the low identity ( 11.6\%) of the Cap, suggesting that there may be a lack of cross-reactivity between the two viruses.

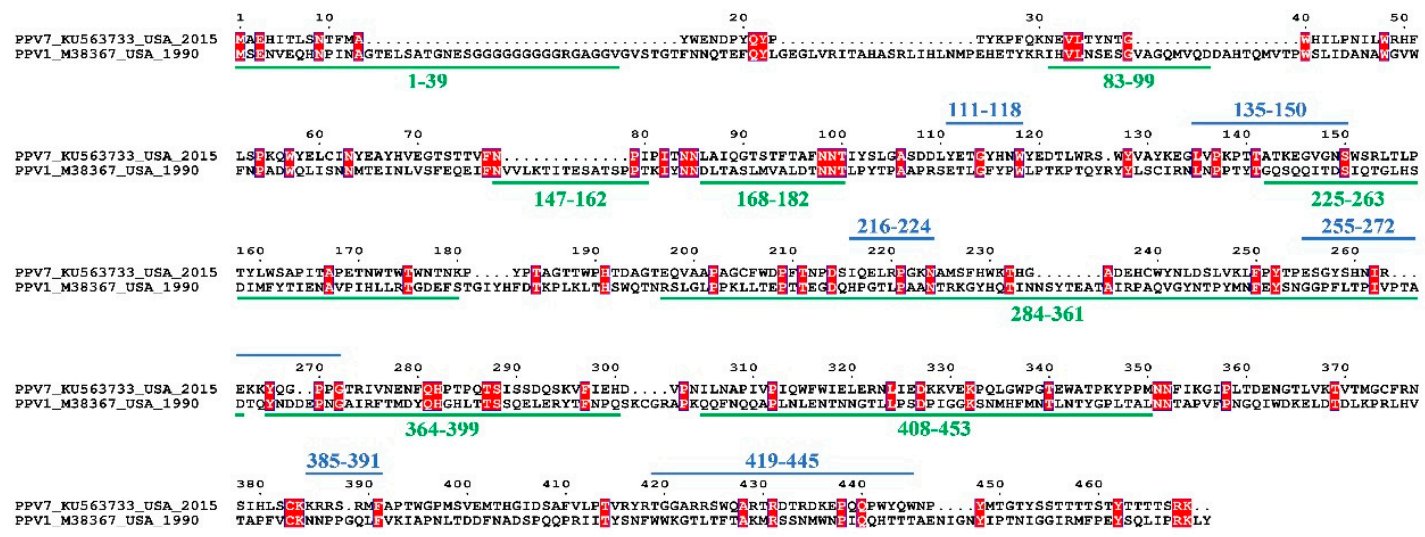

Figure 4. Sequence alignment of predicted PPV7 and identified PPV1 linear B cell epitopes from Cap. Blue lines represent predicted PPV7 B cell epitopes and green lines represent identified PPV1 B cell epitopes.

\subsection{CD8 T Cell Epitopes Prediction and Interaction Study of Predicted Peptides with SLA Alleles}

A total of 12 peptides from the Cap were predicted as CD8 T cell epitopes, whose antigenicities were evaluated with a VaxiJen v2.0 sever (Table S4). The 3D structures of all MHC class-I peptides were modelled via PEP-FOLD3, and a best model for each peptide was used for the subsequent molecular docking with SLA proteins. Among all 12 peptides, 3 peptides were docked to MHC class-I SLA-2*04:02, whereas 6 peptides were docked to MHC class-I SLA-3*04:01. All 9 peptides had high binding affinities. For the 3 peptide-SLA-2*04:02 molecular docking, the binding efficiency of each epitope was evaluated by the global and vdW energies, computed to range from -23.20 to $-54.08 \mathrm{kcal} / \mathrm{mol}$ and -22.21 to $-30.77 \mathrm{kcal} / \mathrm{mol}$, respectively (Table 4$)$. All 3 peptides were predicted to be able to be docked into the groove of the SLA-2*04:02 molecule and form stable hydrogen 
bonds with the residues (within 3.1 $\mathrm{A}$ ) in the groove of the SLA. Furthermore, Asn66 and Asn70 residues from the SLA groove were most abundantly involved in bonding with various peptides (Figure 5A and Figure S5A). In addition, global and vdW energies of the 6 peptide-SLA-3*04:01 dockings ranged from -17.93 to $-44.82 \mathrm{kcal} / \mathrm{mol}$ and -18.96 to $-26.27 \mathrm{kcal} / \mathrm{mol}$, respectively (Table 4 ). Of these 6 peptides, hydrogen bonds $<3 \AA$ were frequently observed in 5 docking complexes to form stable complexes (Figure 5B and Figure S5B). However, the peptide (TAPETNWTW) docked into the groove of SLA-3*04:01 and non-formed hydrogen bonds, whereas the major hydrophobic component interacted with SLA. Notably, 2 overlapped peptides (KRRSRMFAP and RRSRMFAPT) had higher immunogenicity scores (1.2953 and 0.9148), and were strictly conserved in all 59 PPV7 Cap sequences (Table 4).

A

A1

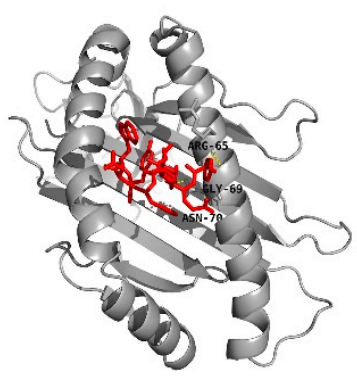

B

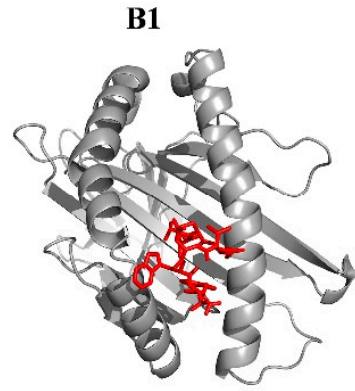

B4

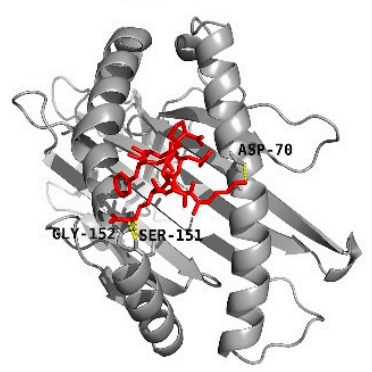

A2

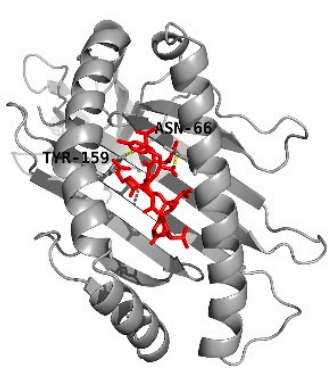

B2

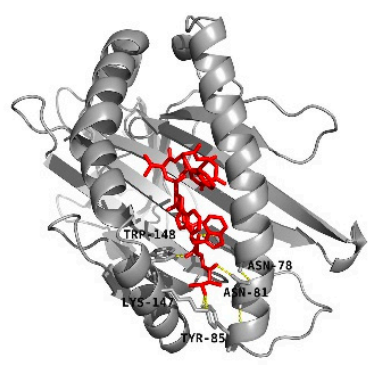

B5

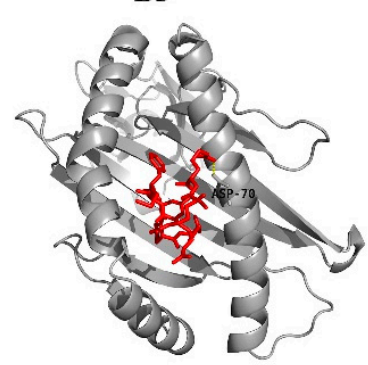

A3

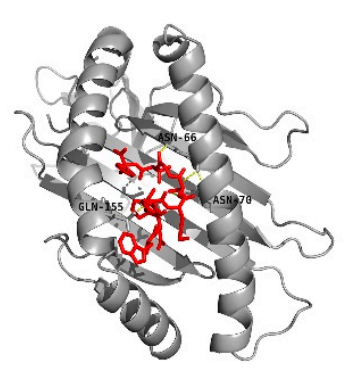

B3

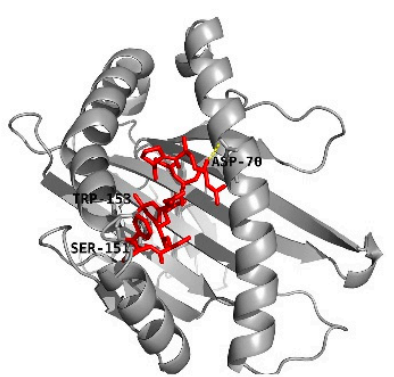

B6

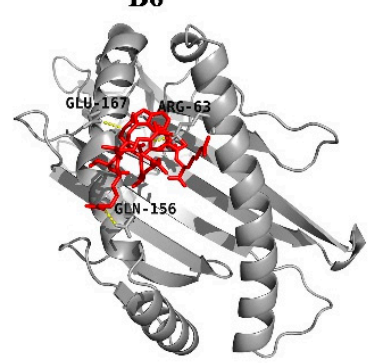

Figure 5. Molecular interaction analysis of PPV7 peptides (shown as red) to SLA-2*0402 (A) and SLA-3*0401 (B) protein (shown as gray). Interacting residues are shown and are consistent with the information in Table 4. 
Table 4. Molecular docking results of SLA-2*0402 and SLA-3*0401 with MHC I peptides.

\begin{tabular}{|c|c|c|c|c|c|c|c|c|c|c|}
\hline No. & Allele & Start & End & Peptide & $\begin{array}{c}\text { Global } \\
\text { Energy } \\
\text { (kcal/mol) }\end{array}$ & $\begin{array}{c}\text { vdW } \\
\text { Energy } \\
\text { (kcal/mol) }\end{array}$ & $\begin{array}{c}\text { H-Bond } \\
\text { Energy } \\
\text { (kcal/mol) }\end{array}$ & $\begin{array}{l}\text { Interacting } \\
\text { Residues }\end{array}$ & $\begin{array}{l}\text { Vaxijen } \\
\text { Score }\end{array}$ & $\begin{array}{c}\text { Identity } \\
\text { (59 Isolates) }\end{array}$ \\
\hline A1 & SLA-2*0402 & 111 & 119 & YETGYHNWY & -26.32 & -22.21 & -1.43 & $\begin{array}{l}\text { Arg65, } \\
\text { Gly69, } \\
\text { Asn70 }\end{array}$ & 0.5659 & $72.88 \%$ \\
\hline A2 & SLA-2*0402 & 409 & 417 & AFVLPTVRY & -23.20 & -24.38 & -1.49 & $\begin{array}{l}\text { Asn66, } \\
\text { Tyr159 }\end{array}$ & 0.7382 & $91.53 \%$ \\
\hline A3 & SLA-2*0402 & 445 & 453 & WNPYMTGTY & -54.08 & -30.77 & -3.19 & $\begin{array}{l}\text { Asn66, } \\
\text { Asn70, } \\
\text { Gln155 }\end{array}$ & 1.2177 & $32.20 \%$ \\
\hline B1 & SLA-3*0401 & 166 & 174 & TAPETNWTW & -25.49 & -21.17 & -0.82 & & 1.4351 & $96.61 \%$ \\
\hline B2 & SLA-3*0401 & 170 & 178 & TNWTWTWNT & -37.42 & -22.35 & -4.1 & $\begin{array}{c}\text { Asn78, } \\
\text { Asn81, } \\
\text { Tyr85, } \\
\text { Lys147, } \\
\text { Trp148 }\end{array}$ & 1.2242 & $33.90 \%$ \\
\hline B3 & SLA-3*0401 & 220 & 228 & RPGKNAMSF & -44.82 & -24.06 & -1.49 & $\begin{array}{l}\text { Asp70, } \\
\text { Ser151, } \\
\text { Trp153 } \\
\text { Asp70 }\end{array}$ & 0.5339 & $91.53 \%$ \\
\hline B4 & SLA-3*0401 & 385 & 393 & KRRSRMFAP & -33.28 & -26.27 & -3.37 & $\begin{array}{l}\text { Ser151, } \\
\text { Gly152 }\end{array}$ & 1.2953 & $100.00 \%$ \\
\hline B5 & SLA-3*0401 & 386 & 394 & RRSRMFAPT & -32.49 & -18.96 & -0.51 & $\begin{array}{l}\text { Asp70 } \\
\text { Arg63, }\end{array}$ & 0.9148 & $100.00 \%$ \\
\hline B6 & SLA-3*0401 & 423 & 431 & RRSWQARTR & -17.93 & -20.24 & -2.17 & $\begin{array}{l}\text { Gln156, } \\
\text { Glu167 }\end{array}$ & 0.6504 & $76.27 \%$ \\
\hline
\end{tabular}

\section{Discussion}

Since the discovery of PPV7 in 2016, most studies have focused on the genetic characterization of individual isolates and epidemiological investigations [13-17]. However, the origin and evolution of this newly emerging PPV are also of interest. To better understand the evolution and genetic relationships of various PPV7 strains, we constructed an MCC tree based on the complete genome sequences of PPV7. We have determined that PPV7 strains have two major clades and may have a common ancestor in approximatively 2004. Furthermore, the PPV7 strains isolated from China are the most likely ancestral strains, based on the collected sequences (Figure 2). To better define PPV7 genotypes, we constructed the NJ and ML trees using NS1 and Cap, respectively, but they did not display similar clusters (data not shown). Thus, a phylogenetic tree analysis did not provide strong evidence for PPV7 genotyping based on current sequences.

The mean evolutionary rates of the PPV7 NS1 gene rate $\left(8.01 \times 10^{-4}\right.$ per site per year) were higher than that of the PPV1 NS1 gene $\left(3.03 \times 10^{-5}\right.$ per site per year) from the previous report [39]. In the PPV7 genotype, the Cap gene had a more rapid evolutionary rate $\left(2.19 \times 10^{-3}\right.$ per site per year) than the NS1 gene $\left(8.01 \times 10^{-4}\right.$ per site per year), which was comparable to the rates of most RNA viruses [40]. In addition, the evolutionary rate of the PPV7 Cap gene was also higher than the rates of the PPV1, PPV2, PPV3 and PPV4 Cap genes $\left(10^{-4}\right.$ per site per year) [39,41]. We also analyzed selective pressures of the PPV7 Cap and NS1 genes. The overall mean difference of dN-dS was -4.122 for the Cap gene, which was higher than that of the NS1 gene (-15.371). Therefore, we inferred that both the Cap and NS1 genes are under purifying selection, whereas the Cap gene undergoes more stringent purifying selection than the NS1 gene. This was supported by the higher evolutionary rate of Cap compared to NS1, and further supported the notion of the correlation between selective pressure and evolutionary rate. PPV7 employs the antigenic variations/shift to resist selective pressures from the host's immune system in the absence of vaccine-induced immune pressure.

The PPV Cap is considered a primary target for eliciting neutralizing antibodies, and has been used for the key antigen of the subunit vaccine against PPV [20,21]. With virtually no information regarding the immune responses against PPV7, computer-based immune-informatics can be used for analysis of antigenic profiles and assist in vaccine development against this newly emerging virus, thereby decreasing cost and time. By selecting the effective antigenic components (epitopes) exposed on the surface, epitope-based vaccines have great potential, as they are capable of inducing strong immune responses in hosts. In this study, we explored potential B cell and CD8 T cell epitopes of PPV7 Cap that may elicit immune responses in the host. After filtering, a total of six linear B cell epitopes 
were predicted in PPV7 Cap located in loops (Figure S3). However, four of the six epitopes were highly variable in residue compositions (Figure S3), although it was not determined whether these epitopes were located on the capsid surface. We tried to predict the 3D structure of PPV7 Cap, but failed due to low identity ( $11.6 \%$ ) with the template of PPV1 Cap (data not shown). The loops of the PPV1 Cap are generally located on the capsid surface, and these loops were the dominant B cell epitope regions and were considered to be important for viral infection and immunogenicity (Figure S4). Amino acid mutations occurred predominantly in these loops [42], and as PPV1 Cap loops are generally located on the capsid surface, these amino acid mutations may influence receptor binding or antigenicity. Thus, high variations of the epitopes located in PPV7 Cap loops may influence receptor binding or antigenicity, if these epitopes are mostly located on the capsid surface; therefore, perhaps PPV7 uses antigenic shift to escape host immune responses. Interestingly, epitope C (IQELMPRKN to IQEL $\underline{R} \underline{\mathrm{G} K N}$ ) had two point mutations (see underlined residues) in isolate PPV7-77, which dramatically decreased the antigenicity score from 0.7908 to 0.2766 . Thereafter, the antigenicity of the peptide containing the double-point mutation in isolate PPV7-77 greatly decreased, which may be one of the strategies of this virus to evade the host's immune responses. Of note, epitope E (KRRSRMF) was highly antigenic (antigenicity score $=1.6044$ ) and strictly conserved among all the isolates of PPV7. Therefore, this potential candidate can be used as a dominant antigen for PPV7 serodiagnosis. Additionally, we predicted several potential CD8 T cell epitopes derived from the PPV7 Cap, and identified nine peptides able be docked onto the SLA with high binding affinities. Based on molecular docking, peptides with potential to SLA and high immunogenicity scores may prove highly immunogenic. Importantly, two conserved overlapped peptides (KRRSRMFAP and RRSRMFAPT) with high immunogenicity scores (1.2953 and 0.9148) should be experimentally tested for the PPV7 vaccine in future studies.

Many parvoviruses, including porcine parvovirus (PPV), human parvovirus B19 (B19V) and human bocavirus 1 (HBoV1), cause infections of their hosts. Among them, B19V is an important human pathogen responsible for a variety of diseases and causes various pathological symptoms, including nonimmune hydrops fetalis and fetal death in pregnant women [43]. Vaccination is the most effective strategy against B19V pathogenesis and infection. Currently, there are two generations of B19V based virus like particles (VLPs) vaccines. The first generation of B19 VLPs consists of two viral structural proteins (VP1 and VP2) produced in the baculovirus expression system, and this induces neutralizing antibodies [44]. These vaccines, produced in insect cells, induced side effects, e.g., reactive symptoms in the host; therefore, baculovirus-based vaccines were abandoned during clinical trials. The second generation of B19 VLPs vaccines, composed of only VP2 protein, are expressed in Saccharomyces cerevisiae [44]. Until now, research for vaccines against B19V has been a huge challenge, due to the unavailability of viral antigens, a good cell line model and a virus-infected animal model. Moreover, there is almost no viremia in most B19V-infected patients when symptoms start to appear. Neutralizing antibodies against B19V are insufficient, emphasizing the importance of developing a vaccine that induces innate and cellular immunity against B19V. The research work of the B19V vaccine provides a further design reference for the PPV7 vaccine.

Based on accumulated evidence, this newly emerging PPV7 has epidemic potential in the global swine population. There is limited information about its epidemiology, transmission, pathogenesis and molecular biology, or indeed, how PPV7 emerges in swine. Regardless, its presence in aborted pig fetuses and its co-infection with PCV2 imply that PPV7 threatens swine herd health security. Notably, the substitution rate of PPV7 was higher than PPV1-4, which may enable PPV7 to adapt to various environmental conditions and cause a substantial threat to the swine herd. Thus, a vaccine against PPV7 is needed to control this emerging virus infection. Inactivated vaccines against PPV1 have been used for 30 years because they hindered or reduced virus transmission. Low homology between PPV7 Cap and PPV1 suggested the PPV1 vaccine strains were not closely matched with PPV7 strains. Novel vaccine formulations containing PPV1 and the newly circulating strains PPV7 may overcome some potential weaknesses of current vaccines, perhaps increasing vaccine efficacy. 


\section{Conclusions}

In conclusion, this study has provided evidence on the evolutionary dynamics of PPV7. We concluded that PPV7 has a more rapid evolutionary rate than other PPV genotypes. In addition, antigenic profiles of the PPV7 Cap were revealed by immunoinformatics, and there were indications that PPV7 evades the host's immune responses via antigenic shifts during virus evolution, in the absence of vaccine-induced immune pressure. In addition, these potential B cell epitopes identified in this study may serve as antigens for PPV7 vaccine or for serological diagnosis, with further experiments warranted.

Supplementary Materials: The following are available online at http://www.mdpi.com/2076-393X/8/3/359/s1, Figure S1: Phylogenetic analysis of viruses in the Parvoviridae family, Figure S2: Secondary structure analysis of PPV7 Cap via PSIPRED, Figure S3: Sequence alignment of PPV7 Cap, Figure S4: Mapping the location of identified PPV1 B cell epitope regions on 3D structure (PDB ID: 1K3V) of capsid (A) and Cap monomer (B,C), Figure S5: 2D graphical representation of molecular interaction analysis of MHC class-I alleles binding peptides to SLA-2*0402 (A) and SLA-3*0401 (B) protein, Table S1: Detailed information of PPV7 strain sequences used in this study, Table S2: Detailed information of reference Parvoviridae sequences used in this study, Table S3: Identified linear B cell epitopes in PPV1 Cap deposited in IEDB database, Table S4: CD8 T cell epitopes prediction of PPV7 Cap.

Author Contributions: Conceptualization, D.W., N.W.; methodology, D.W., J.M.; software, D.W., J.M.; validation, D.W., J.M.; formal analysis, D.W., J.M.; investigation, D.W.; resources, D.W.; data curation, D.W.; writing-original draft preparation, D.W., N.W.; writing-review and editing, N.W., Y.Y.; visualization, D.W.; supervision, N.W., Y.Y.; project administration, N.W., Y.Y.; funding acquisition, D.W., N.W. All authors have read and agreed to the published version of the manuscript.

Funding: This research was funded by Hunan Provincial Natural Science Foundation of China, grant number 2018JJ2177; Double first-class construction project of Hunan Agricultural University, grant number SYL2019048 and Postgraduate Research and Innovation Project of Hunan Province, grant number CX2018B394.

Conflicts of Interest: The authors declare no conflict of interest.

\section{References}

1. Cotmore, S.F.; Agbandje-Mckenna, M.; Chiorini, J.A.; Mukha, D.V.; Davison, A. The family Parvoviridae. Arch. Virol. 2013, 159, 1239-1247. [CrossRef] [PubMed]

2. Palinski, R.M.; Mitra, N.; Hause, B.M. Discovery of a novel Parvovirinae virus, porcine parvovirus 7, by metagenomic sequencing of porcine rectal swabs. Virus Genes 2016, 52, 564-567. [CrossRef] [PubMed]

3. Streck, A.F.; Canal, C.W.; Truyen, U. Molecular epidemiology and evolution of porcine parvoviruses. Infect. Genet. Evol. 2015, 36, 300-306. [CrossRef] [PubMed]

4. Mayr, A.; Mahnel, H. Cultivation of hog cholera virus in pig kidney cultures with cytopathogenic effect. Zent. Bakteriol Orig 1965, 195, 157-166.

5. Mengeling, W.L.; Lager, K.M.; Vorwald, A.C. The effect of porcine parvovirus and porcine reproductive and respiratory syndrome virus on porcine reproductive performance. Anim. Reprod. Sci. 2000, 60, $199-210$. [CrossRef]

6. Hueffer, K.; Parrish, C. Parvovirus host range, cell tropism and evolution. Curr. Opin. Microbiol. 2003, 6, 392-398. [CrossRef]

7. Hijikata, M.; Abe, K.; Win, K.M.; Shimizu, Y.K.; Yoshikura, H. Identification of new parvovirus DNA sequence in swine sera from Myanmar. Jpn. J. Infect. Dis. 2002, 54, 244-245.

8. Lau, S.K.P.; Woo, P.C.Y.; Tse, H.; Fu, C.T.Y.; Au, W.-K.; Chen, X.-C.; Tsoi, H.-W.; Tsang, T.H.F.; Chan, J.S.Y.; Tsang, D.N.C.; et al. Identification of novel porcine and bovine parvoviruses closely related to human parvovirus. J. Gen. Virol. 2008, 89, 1840-1848. [CrossRef]

9. Cheung, A.K.; Wu, G.; Wang, D.; Bayles, D.O.; Lager, K.M.; Vincent, A.L. Identification and molecular cloning of a novel porcine parvovirus. Arch. Virol. 2010, 155, 801-806. [CrossRef]

10. Xiao, C.T.; Giménez-Lirola, L.G.; Jiang, Y.-H.; Halbur, P.G.; Opriessnig, T.; Elankumaran, S. Characterization of a novel porcine parvovirus tentatively designated PPV5. PLoS ONE 2013, 8, e65312. [CrossRef]

11. Ni, J.; Qiao, C.; Han, X.; Han, T.; Kang, W.; Zi, Z.; Cao, Z.; Zhai, X.; Cai, X. Identification and genomic characterization of a novel porcine parvovirus (PPV6) in China. Virol. J. 2014, 11, 203. [CrossRef]

12. Anne-Lie, B.M.; Ye, X.; Caroline, F.; Per, W.; Mikael, B. Characterisation of the virome of tonsils from conventional pigs and from specific pathogen-free pigs. Viruses 2018, 10, 382. 
13. Miłek, D.; Woźniak, A.; Stadejek, T. The detection and genetic diversity of novel porcine parvovirus 7 (PPV7) on Polish pig farms. Res. Vet. Sci. 2018, 120, 28-32. [CrossRef] [PubMed]

14. Xing, X.; Zhou, H.; Tong, L.; Chen, Y.; Sun, Y.; Wang, H.; Zhang, G. First identification of porcine parvovirus 7 in China. Arch. Virol. 2018, 163, 209-213. [CrossRef] [PubMed]

15. Wang, Y.; Yang, K.-K.; Wang, J.; Wang, X.-P.; Zhao, L.; Sun, P.; Li, Y.-D. Detection and molecular characterization of novel porcine parvovirus 7 in Anhui province from Central-Eastern China. Infect. Genet. Evol. 2019, 71, 31-35. [CrossRef] [PubMed]

16. Wang, W.; Cao, L.; Sun, W.; Xin, J.; Zheng, M.; Tian, M.; Lu, H.; Jin, N. Sequence and phylogenetic analysis of novel porcine parvovirus 7 isolates from pigs in Guangxi, China. PLoS ONE 2019, 14, e0219560. [CrossRef]

17. Ouh, I.-O.; Park, S.; Lee, J.-Y.; Song, J.Y.; Cho, I.-S.; Kim, H.-R.; Park, C.-K. First detection and genetic characterization of porcine parvovirus 7 from Korean domestic pig farms. J. Vet. Sci. 2018, 19, 855-857. [CrossRef]

18. Da Silva, M.S.; Budaszewski, R.F.; Weber, M.N.; Cibulski, S.P.; Paim, W.P.; Mósena, A.C.S.; Canova, R.; Varela, A.P.M.; Mayer, F.Q.; Pereira, C.W.; et al. Liver virome of healthy pigs reveals diverse small ssDNA viral genomes. Infect. Genet. Evol. 2020, 81, 104203. [CrossRef]

19. Chung, H.-C.; Nguyen, V.-G.; Huynh, T.-M.-L.; Park, Y.-H.; Park, K.-T.; Park, B.-K. PCR-based detection and genetic characterization of porcine parvoviruses in South Korea in 2018. BMC Vet. Res. 2020, 16, 113. [CrossRef]

20. Antonis, A.F.G.; Bruschke, C.J.M.; Rueda, P.; Maranga, L.; Casal, J.I.; Vela, C.; Hilgers, L.A.T.; Belt, P.B.G.M.; Weerdmeester, K.; Carrondo, M.J.T. A novel recombinant virus-like particle vaccine for prevention of porcine parvovirus-induced reproductive failure. Vaccine 2006, 24, 5481-5490. [CrossRef]

21. Ji, P.; Liu, Y.; Chen, Y.; Wang, A.; Jiang, D.; Zhao, B.; Wang, J.; Chai, S.; Zhou, E.; Zhang, G. Porcine parvovirus capsid protein expressed in Escherichia coli self-assembles into virus-like particles with high immunogenicity in mice and guinea pigs. Antivir. Res. 2017, 139, 146-152. [CrossRef]

22. Saade, G.; Deblanc, C.; Bougon, J.; Marois-Créhan, C.; Fablet, C.; Auray, G.; Belloc, C.; Leblanc-Maridor, M.; Gagnon, C.A.; Zhu, J.; et al. Coinfections and their molecular consequences in the porcine respiratory tract. Vet. Res. 2020, 51, 80. [CrossRef] [PubMed]

23. Miłek, D.; Woźniak, A.; Podgórska, K.; Stadejek, T. Do porcine parvoviruses 1 through 7 (PPV1-PPV7) have an impact on porcine circovirus type 2 (PCV2) viremia in pigs? Vet. Microbiol. 2020, 242, 108613. [CrossRef]

24. Kumar, S.; Stecher, G.; Tamura, K. MEGA7: Molecular evolutionary genetics analysis version 7.0 for bigger datasets. Mol. Biol. Evol. 2016, 33, 1870-1874. [CrossRef] [PubMed]

25. Drummond, A.J.; Rambaut, A. BEAST: Bayesian evolutionary analysis by sampling trees. BMC Evol. Biol. 2007, 7, 214. [CrossRef] [PubMed]

26. Kosakovsky, P.S.L.; Frost, S.D.W. Not so different after all: A comparison of methods for detecting amino acid sites under selection. Mol. Biol. Evol. 2005, 22, 1208-1222. [CrossRef]

27. Ben, M.; Sasha, M.; Amandla, M.; Thomas, W.; Daniel, S.; Pond, S.L.K.; Konrad, S. FUBAR: A fast, unconstrained bayesian AppRoximation for inferring selection. Mol. Biol. Evol. 2013, 30, 1196-1205.

28. Murrell, B.; Wertheim, J.O.; Moola, S.; Weighill, T.; Scheffler, K.; Pond, S.L.K. Detecting individual sites subject to episodic diversifying selection. PLoS Genet. 2012, 8, e1002764. [CrossRef]

29. Hughes, A.L.; Nei, M. Nucleotide substitution at major histocompatibility complex class II loci: Evidence for overdominant selection. Proc. Natl. Acad. Sci. USA 1989, 86, 958-962. [CrossRef]

30. Buchan, D.W.A.; Minneci, F.; Nugent, T.; Bryson, K.; Jones, D.T. Scalable web services for the PSIPRED Protein Analysis Workbench. Nucleic Acids Res. 2013, 41, W349-W357. [CrossRef]

31. Doytchinova, I.A.; Flower, D.R. VaxiJen: A server for prediction of protective antigens, tumour antigens and subunit vaccines. BMC Bioinform. 2007, 8. [CrossRef] [PubMed]

32. Pedersen, L.E.; Harndahl, M.; Nielsen, M.; Patch, J.R.; Jungersen, G.; Buus, S.; Golde, W.T. Identification of peptides from foot-and-mouth disease virus structural proteins bound by class I swine leukocyte antigen (SLA) alleles, SLA-1*0401 and SLA-2*0401. Anim. Genet. 2013, 44, 251-258. [CrossRef] [PubMed]

33. Pedersen, L.E.; Rasmussen, M.; Harndahl, M.; Nielsen, M.; Buus, S.; Jungersen, G. A combined prediction strategy increases identification of peptides bound with high affinity and stability to porcine MHC class I molecules SLA-1*04:01, SLA-2*04:01, and SLA-3*04:01. Immunogenetics 2016, 68, 157-165. [CrossRef] 
34. Gutiérrez, A.H.; Martin, W.D.; Bailey-Kellogg, C.; Terry, F.; Moise, L.; Groot, A.S.D. Development and validation of an epitope prediction tool for swine (PigMatrix) based on the pocket profile method. BMC Bioinform. 2015, 16, 290. [CrossRef] [PubMed]

35. Alexis, L.; Pierre, T.; Julien, R.; Marek, V.; Philippe, D.; Pierre, T.J.N.A.R. PEP-FOLD3: Faster de novo structure prediction for linear peptides in solution and in complex. Nucleic Acids Res. 2016, 44, W449-W454.

36. Schneidman-Duhovny, D.; Inbar, Y.; Nussinov, R.; Wolfson, H.J. PatchDock and SymmDock: Servers for rigid and symmetric docking. Nucleic acids Res. 2005, 33, W363-W367. [CrossRef] [PubMed]

37. Andrusier, N.; Nussinov, R.; Wolfson, H.J. FireDock: Fast interaction refinement in molecular docking. Proteins Struct. Funct. Bioinform. 2007, 69, 139-159. [CrossRef]

38. Efrat, M.; Dina, S.D.; Nelly, A.; Ruth, N.; Wolfson, H.J. FireDock: A web server for fast interaction refinement in molecular docking. Nucleic Acids Res. 2008, 36, W229-W232.

39. Ren, X.; Tao, Y.; Cui, J.; Suo, S.; Cong, Y.; Tijssen, P. Phylogeny and evolution of porcine parvovirus. Virus Res. 2013, 178, 392-397. [CrossRef]

40. Jenkins, G.M.; Rambaut, A.; Pybus, O.G.; Holmes, E.C. Rates of molecular evolution in RNA viruses: A quantitative phylogenetic analysis. J. Mol. Evol. 2002, 54, 156-165. [CrossRef]

41. Cadar, D.; Lo"rincz, M.; Kiss, T.; Novosel, D.; Podgorska, K.; Becskei, Z.; Tuboly, T.s.; Csa'gola, A. Emerging novel porcine parvoviruses in Europe: Origin, evolution, phylodynamics and phylogeography. J. Gen. Virol. 2013, 94, 2330-2337. [CrossRef] [PubMed]

42. Streck, A.F.; Bonatto, S.L.; Homeier, T.; Souza, C.K.; Truyen, U. High rate of viral evolution in the capsid protein of porcine parvovirus. J. Gen. Virol. 2011, 92, 2628-2636. [CrossRef] [PubMed]

43. Qiu, J.; Söderlund-Venermo, M.; Young, N.S. Human parvoviruses. Clin. Microbiol. Rev. 2017, 30, 43-113. [CrossRef] [PubMed]

44. Das, P.; Chatterjee, K.; Chattopadhyay, N.R.; Choudhuri, T. Evolutionary aspects of Parvovirus B-19V associated diseases and their pathogenesis patterns with an emphasis on vaccine development. Virusdisease 2019, 30, 32-42. [CrossRef]

(C) 2020 by the authors. Licensee MDPI, Basel, Switzerland. This article is an open access article distributed under the terms and conditions of the Creative Commons Attribution (CC BY) license (http://creativecommons.org/licenses/by/4.0/). 